\title{
Understanding théoro-empirical correlation of CSR: ASP Model
}

\author{
Benabdeslam Rim \\ Phd Student at University Mohammed V Agdal Rabat Morocco and Phd student at University Paul Valéry \\ Montpellier III France
}

\begin{abstract}
It is wise before the idea of building a model, read and make sure it is really necessary to do first. See you in the event that the literature provides us with competing models and theories. It is then necessary to proceed by reading, in other words to test each of these models to retain more or less specifically or to see everyone's contribution to the knowledge of the phenomenon.

The approach taken tries to answer the obvious question that arises every researcher on the evaluation of the models and the choice between them. A question that lies at the heart of the unresolved debate between rationalism and relativism. These two currents oppose.

When models are competing penchant for one is not the fruit of justification by experience statements constituting the theory or finding of a logical reduction of the theory to experiment. Popper (1973) proposes to adopt the model that "defends the better," that is to say the one that seems to be more representative of reality. And we follow this approach in the sense that building our own model based on an evaluation of the existing. We call our ASP model.
\end{abstract}

Keywords: corporate social responsibility, ASP Model,

\section{Introduction}

Theoretical models of PSE were used mainly in research and descriptive analysis but rarely as such as the metering mode. The institutionalization of CSR refers to driving measurable and therefore shares already, which makes it highly experienced limited practical operationalization.

We try to draw the guidelines of established models and involve measures which make a CSR model applicable in a more pragmatic and scientific logic.

\section{Methodology}

We perform a comparative analysis of existing models and derive a short summary In contrast to this comparative analysis, findings emerge:

Each author describes a particular approach:

$\Longrightarrow$ Principles/Issues management/Responsiveness: Carroll

$\Longrightarrow$ Principles/Responsiveness/Policies: Watrick et Cochran

$\Longrightarrow$ Principles/Responsiveness/Results: Wood

- The "social responsiveness" dimension is perceived differently for each of these authors

- Interrelation firm / company

From these three observations, we can comment on the need for some combination of the various parameters listed in the models. Therefore, we use a 4-phase approach: principle / Responsiveness / Policies / results.

In this sense the social responsibility principles are divided into three levels: institutional, organizational and individual. This means that all decisions are CSR involve all stakeholders and incorporated into the overall strategy of the firm to be broken down into operational and strategic goals and serving as the basis of reaction for each individual participant to its implementation.

Responsiveness is the design of processes to be deployed to implement the agreed strategy. Policy it differs receptivity, being a form of dissemination of the strategy and thus is a projection and continuity of receptivity base. The results are the end point of the approach but nevertheless represents the starting point of a new action to be taken. After dissemination of the strategy, it must be maintained and continuously evaluated. The results are not the result of the strategy but also the perseverance and domination continually.

Our approach will be called the four-time 4D and will be presented in the next section.

From the above, the word strategy comes back several times and denotes a large extent given to it. Therefore, we believe it should be a pillar entire share aspects of CSR. According to this principle, companies are required to act in accordance with the formal procedures to meet their economic and social commitments. It also 
legitimizes the outsourcing or subcontracting social actions by engaging external entities or coordination mechanisms with the market. This legitimacy gives companies the right to act on a perceived power through a shared operability with external or internal actors thus showing the concept of stakeholder environment.

This fits perfectly with the principles with their three levels of analysis (institutional, organizational, and individual) The inter-firm / company confirms the nature of involvement of stakeholders and requires the presence of a cross-pillar that serves as a common ground of understanding and communication interface

As for responsiveness, it is viewed by Wood as an achievable goal. This current view that responsiveness is tangible and it could replace social responsibility. Watrick and Cochran for their view that responsiveness does not necessarily mean being responsible and that social responsibility generally refers to earnings, while receptivity process. From this follows two complementary concepts that contribute to the definition of social performance. The risk of substituting the concept of social responsibility that social responsiveness is to perceive the RS as a public relations exercise wheels

\section{Discussion}

This mode of proposed engineering is based on three axes called 3M: Mutation, Method and Medium. Its implementation however is done in 4 phases nicknamed 4D: Decision Arrangement Dissemination and Domination. We will see that the results obtained may be approved had sought against the criteria but in terms of creating potential or measurable from corporate earnings immediate results.

To present in more detail the model, we perform a sequential breakdown of the process of implementation thereof

\section{The Process Model Axial}

Axial process is the progressive approach that integrates the company within involving the top management and the responsibility centers and the various entities that are connected simultaneously. It's an approach based on an implementation of the strategic management of CSR in three stages.

\subsection{The three pillars of the establishment of the strategic management of CSR \\ 1.1.1 Method and behavioral attitudes}

This axis is the basis for strategic development choices and procedures deployed for this purpose and therefore it involves a strategic review of any changes to take technologically and organizationally. It is based on the political speeches and active behaviors of leaders who it belongs the authority to establish such a step, to follow and to involve all stakeholders in the organization. Interaction with two axes and is off.

This line joins the transverse pillar in the sense that it is related to the implementation of each decision. The company tends through a "synchronized decentralization" to link the decision to the responsibility center from which it emanates consistent with the actions of other responsibility centers but also in consistent with the overall business strategy .

\subsubsection{Resources deployed}

Establish a management or incorporate a new social application guidance refers to a number of ways, each of which participates in the implementation of the strategy decided in advance and allows to carry out effectively the change to be made.

\subsubsection{Mutation}

The mutation is a metamorphosis that involves the establishment of an innovative approach through appropriate tools and following a decision taken unanimously.

Thus, this pin is introduced in three stages:

\section{Identification $>$ Conception $>$ Application}

\section{Phases of the model " $4 \mathrm{D}$ "}

The axial process itself cannot be achieved randomly. The company in its definition crafted a detailed plan from simple decision to the actual performance by exploiting certain procedures in force in the classical management and district planners.

\subsection{To decide policy CSR}

Driving a change is certainly not an easy process especially since the entire operation of the company can be reviewed. Alongside the fierce competition encourages companies to innovate and make concrete actions in these innovation policies. 
Making good decisions is of listing the pros and cons of CSR. In this sense in order to make a good decision, leaders begin this phase by a period of reflection before a decision and therefore proceed to the confirmation.

This part will then comprise the first phase of our proposed model, which is to the decision of setting up a MS CSR.

\subsection{To Have A CSR}

The provision of a CSR or its layout indicates the way that should apply to CSR. It does not determine the method deployed for this purpose but to actually implement this method raised in the decision phase.

This is a step to take action and move from a conventional management to strategic management of CSR. For the enterprise, the process of implanting said within it and to make changes in his actions.

\subsection{Dissemination Csr In Business}

Disseminate CSR in a company's returns to involve all stakeholders, and its successful implementation.

Success depends heavily on the performance of the company's management. An approach succeeds best when its leaders manage to integrate employees.

In other words, it is to highlight the dialogue, but dialogue delegation as pillars of management in which the progression of skills and career development include personal projects, and relationships are based on mutual respect and recognition.

\subsection{Domination.}

During this last phase, the company is in the process of evaluation and sustainability. In other words, the company must ask the question of the interest it has been removed by the implementation of a strategy or strategic management of CSR. Evaluation is a crucial step. One always wonders why did we need to evaluate? Or in the event that we believe that evaluation is an integral step in a process of change one wonders then how do we must make this assessment?

\section{The pillars of the model " $4 \mathrm{P}$ "}

In these four steps and axial process, we aim to implement a more strategic management that incorporates the principles of CSR.

The pillars of the model are known as the number three; economic, social and environmental. Various authors prefer to rename CSR social responsibility of the company and accordingly believes that the society is a fundamental pillar in its execution.

Following our qualitative analysis and theoretical reading, we considered a new pillar equally important and embraces the concept of cross and institutionalization of CSR practices.

\subsection{Environmental Pillar}

This pillar includes all the environmental activities: These include the fight against climate change, the economic management of resources and waste recycling and biodiversity.

For firms, the establishment of an environmental strategy is cardinal because it allows both to sustain its activities in the face of dwindling resources, accelerating climate change and rising energy prices and reduce the impact of its activities on the environment and thus be part of an ethical approach.

\subsection{Economic Pillar}

This component reaffirms the relationship between the company and its customers, suppliers and civil society. Indeed, in this aspect it is to reconcile the company's viability with environmental and social actions by appealing to external stakeholders

The challenges of a responsible economy are numerous and often related to one of the environmental and social pillars, or both at once. Seek alternative methods require the involvement of stakeholders: governments, NGOs, unions, customers

In this sense and according to the major customers of the company, it occurs in very specific fields and agrees to comply with well-defined terms.

\subsection{Social Pillar}

Social CSR strategy is not only to administrative procedures in terms of human resource management, but it covers a variety of functions such as working conditions and human capital development

The definition of an HR strategy revolves around the win-win principle, because satisfied employees working conditions are more receptive to change and remain more efficient. In this sense, several possibilities 
can be envisaged. These include the creation of a conducive working environment, the fight against discrimination, flexible working hours tailored to the individual needs of staff, work motivation, training .

\subsection{Strategic Pillar}

Taking a strategic CSR is a decision for the top management of the company in question. The reasons are varied and differ considerably from one context to another. The term context, all elements that distinguish one company from another, the industry, the settlement area, the size .

CSR policies involve integration into the managerial process by evaluating the strategy and medium and long term investments. For example, we include policies to reduce water consumption which can be a cost factor but also gain by being rewarded with reduced expense by changing production conditions.

The implementation of CSR policies through a development of the valuation of the company and an active integration of a set of processes and actors. This new vision adopts a transversal deployment following the implementation of CSR in all its stages and all its components. This policy results in relevant and comparable from one period to another non-financial data.

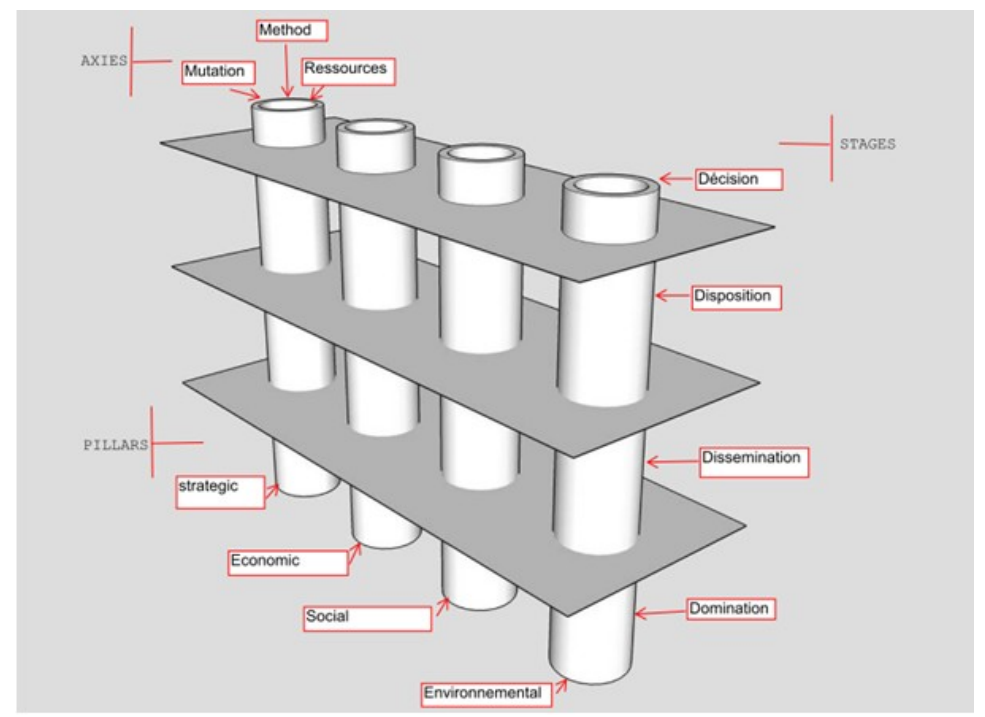

\section{Conclusion}

We called our ASP because it includes the axes A, S steps and pillars P.

This is a model that takes into account the literature review and field research carried out in a Moroccan context;

However, the establishment of a model is not sufficient to decide on the relevance of the latter, so a test of the model is essential.

\section{References}

[1] Carroll, A.B. (1994). "Social issues in management Research". Business and society, 33 (1), 5-29

[2] Carroll, A.B. (1999). "Corporate social responsibility". Business and society, 38 (3), 268-295

[3] Carroll, A.B. (1979). “A three-dimensional conceptual model of corporate performance". Academy of management Review, 4 (4), 497-505

[4] Clarkson, M.B.E. (1991). "Defining, evaluating, and managing corporate social performance: The stakeholder management model." In: Post, J.E. (ed.), Research in corporate social performance and policy, 331-358

[5] Clarkson, M.B.E. (1995). "A stakeholder framework for analyzing and evaluating corporate social performance." Academy of management review, 20 (1), $92-117$

[6] EL malki Tarik (2010), La responsabilité sociale des enterprises: le cas du Maroc

[7] J-p.Gond et J.Igalens (2008), La responsabilité sociale de l'entreprise

[8] Savall H. et Zardet V. (1995), Maîtriser les coûts et les performances cachés, 1995, Economica 